\title{
Foxtail Millet Stress Associated Protein Gene SiSAP4 Enhances Drought Stress Tolerance in Transgenic Arabidopsis
}

\author{
Wenlu Li ${ }^{1}$, Liguang Zhang ${ }^{1}$, Faheeda Soomro ${ }^{2,3}$, Pingyi Guo ${ }^{1}$, Xiangyang Yuan $^{1^{*}}$ and Yixue Wang ${ }^{2 *}$ \\ ${ }^{1}$ College of Agronomy, Shanxi Agricultural University, Jinzhong 030801, China \\ ${ }^{2}$ College of Life Sciences, Shanxi Agricultural University, Jinzhong 030801, China \\ ${ }^{3}$ International Center for Chemical and Biological Sciences, University of Karachi, Karachi 75270, Pakistan \\ *For correspondence: yuanxiangyang200@163.com; ciciwyx@163.com \\ Received 14 September 2020; Accepted 22 October 2020; Published 10 January 2021
}

\begin{abstract}
Abiotic stresses like drought affect plant growth and crop yield with climate change worsening. Stress associated proteins (SAPs), as the zinc finger proteins with A20/AN1 domain, play an important role in regulating abiotic stress response. As a typical summer dryland grain crop in the north of China, foxtail millet has the characteristics of drought resistance, making it a valuable resource for anti-stress gene exploitation and utilization. In this study, SiSAP4 gene was cloned from foxtail millet variety Yugu 1. Analysis showed that SiSAP4 gene was expressed in roots, stems and leaves at seedling stage, and the highest expression level was detected in leaves. Expression patterns under different stress conditions showed that expression level of SiSAP4 gene was significantly up-regulated under drought stress, suggesting it may be involved in drought stress response. Subcellular localization indicated that SiSAP4 was present in the nucleus and cytoplasm. It was revealed that SiSAP4 had no function in transcriptional activation in the yeast system. Overexpression of SiSAP4 in transgenic Arabidopsis resulted in enhanced tolerance to drought stress, which was simultaneously demonstrated by increased expression of a broad range of stress response genes. Based on those results, SiSAP4 has the potential to be used in transgenic breeding to improve drought stress tolerance in other crops. (C) 2021 Friends Science Publishers
\end{abstract}

Keywords: Foxtail millet; SiSAP4; Drought tolerance; Transgenic Arabidopsis

\section{Introduction}

Plants are mostly affected with abiotic stresses such as high temperatures, waterlogging, salt and drought stress. During the process of evolution, plants formed complex regulation network mechanism in response to environmental challenges. Plants can be regulated at molecular and cellular levels to survive adverse environmental events (Hirayama and Shinozaki 2010; Shi et al. 2015). This regulatory network relies on transcriptional factors to activate downstream target genes in response to environmental stimulus (Zhang et al. 2012; Chakraborty et al. 2015). Zinc finger proteins contain numerous functional proteins that play major roles as transcriptional factors, protein modification enzymes and RNA-binding proteins that protect cells against environmental stresses (Zhang et al. 2014; Baek et al. 2015; Wang et al. 2019; Han et al. 2020). Stress associated proteins (SAPs) belong to A20/AN1 zinc finger proteins involved in pathways for plant growth and development and in abiotic stress tolerance.

The SAP gene family is broadly present across plant species. Until now, many $S A P$ genes have been identified. OsiSAPl was the first member among this gene family to be studied (Giri et al. 2013). OsiSAP1 overexpression has been found to enhance the resistance of transgenic tobacco to high salinity, low temperature and drought condition during germination and seedling stages (Mukhopadhyay et al. 2004). It has been shown that OsiSAP8 can be induced by a variety of adverse stresses, namely heat, cold, salt, desiccation, submergence, wounding and heavy metals. Overexpression of OsiSAP8 showed no significant reduction in rice yield with high salinity and drought stresses at flowering stage (Kanneganti and Gupta 2008). AtSAP5 can improve the tolerance of transgenic cotton to heat stress by regulating the expression of genes related to water deficit and heat stress protecting the photosystem II complex in photosynthesis (Hozain et al. 2012). TaSAP5 can function as E3 ubiquitin ligase, which can ubiquitinize and degrade DRIP protein to increase the accumulation of DREB2A protein, activate the expression of downstream genes, and improve the drought resistance of wheat (Zhang et al. 2017). In addition, SAP genes have been reported in maize, sorghum, tomato, medicago, banana, aeluropus littoralis and other crops, where expression responses under stress conditions were determined and functional validation 
using transgenic systems performed (Solanke et al. 2009; Saad et al. 2010; Xuan et al. 2011; Charrier et al. 2012; Sreedharan et al. 2012; Wang et al. 2013).

Foxtail millet (Setaria italica L.) originated from northern China and has been an important dryland grain crop. Foxtail millet not only has the characteristics of strong adaptability to drought and infertility, but also has a small genome size, genetic diversity and short life cycle. Therefore, it can be a valuable resource for abiotic stress resistant gene exploration (Muthamilarasan and Prasad 2015; Yang et al. 2020). At present, the research on functional genomic data of foxtail millet including the annotation and functional characterization of genes involved in abiotic stress responses has been not analyzed. In this study, SiSAP4 was cloned from Yugu 1. Expression profile of this gene was analyzed in foxtail millet and the function validated through transfer of the gene to Arabidopsis.

\section{Materials and Methods}

\section{Plant treatment and growing conditions}

Foxtail millet variety "Yugu 1" was used in this study. The millet seeds were planted in vermiculite: nutrient soil 1:1 for three weeks at $23^{\circ} \mathrm{C}$ with a $16 / 8 \mathrm{~h}$ (light/dark) photoperiod in the chamber. The leaves, stems and roots of the seedlings were taken and utilized to analyze the expression levels of the target gene in different tissues. To analyze the expression levels of target gene in variable stress conditions, millet seedlings with uniform growth at three weeks were selected for stress treatments (Min et al. 2013). These millet seedlings were exposed to the following stress treatments: $6 \%$ PEG 6000, $100 \mathrm{mM} \mathrm{NaCl}$ and low temperature $\left(4^{\circ} \mathrm{C}\right)$. Leaf samples were collected at $0.5,1,3,6,9,12$ and $24 \mathrm{~h}$ after stress treatment, respectively. All samples were stored at $-80^{\circ} \mathrm{C}$ at once.

Transgenic studies were conducted on Arabidopsis thaliana ecotype Col-0 with the chamber condition as: temperature $-23^{\circ} \mathrm{C}$; photoperiod $-16 / 8 \mathrm{~h}$ (light/dark) and relative humidity $-65 \%$. To study the expression pattern of water deficit stress response genes both wild type (WT) and transgenic Arabidopsis were grown on MS medium supplemented with $250 \mathrm{mM}$ mannitol for $3 \mathrm{~h}$.

\section{Isolation of SiSAP4 gene and sequence analysis}

The full-length cDNA of SiSAP4 was amplified using primers SiSAP4 (forward primer, 5'AGTAGTCATGGAACACAAGG -3'; reverse primer, 5'CTTGCAGATCACAACCCATC -3'). pEASY-Blunt vectors (TransGen, Beijing) were used for PCR product ligation after purification. After successful transformation, the positive clones were picked for sequencing. The amino acid composition, molecular weight and isoelectric point of SiSAP4 were predicted by online analysis software
ProtParam tool (https://web.expasy.org/protparam/). The protein sequence of SiSAP4 was used as query in a BLASTP program by collecting highly similar sequences to study relationship between SiSAP4 with other family members from NCBI website. Alignment of sequences was performed by DNAMAN to check similarity and phylogenetic tree was built by neighborjoining method with 1000 bootstrap replicates using MEGA5.0 (Tamura et al. 2011).

\section{Quantitative real-time PCR}

Total RNA was isolated from foxtail millet seedlings and Arabidopsis plants using RNAprep Pure Plant Kit (Tiangen, Beijing) as manufacturer's instructions. The cDNA was synthesized from RNA template after treatment with DNaseI using Fast Quant RT Kit (Tiangen, Beijing). To check the level of gene expression quantitative real-time PCR (qRT-PCR) was done. The internal control SiActin (forward primer, 5'- GGCAAACAGGGAGAAGATGA 3'; reverse primer, 5'- GAGGTTGTCGGTAAGGTCACG -3') and AtActin2 (forward primer, 5'AGCACTTGCACCAAGCAGCATG-3’; reverse primer, 5'-ACGATTCCTGGACCTGCCTCATC-3') were utilized to determine the relative transcript level of target genes in the Arabidopsis and foxtail millet. The qRT-PCR was done in three replicates with an ABI Prism 7500 system consuming the SYBR Green Master Mix kit (TaKaRa, Japan). The relative gene expression levels were calculated by the $2^{-\triangle \Delta^{C T}}$ method (Schmittgen and Livak 2008).

\section{Subcellular localization of SiSAP4 protein}

Agrobacterium mediated transformation was performed in tobacco leaves using strain GV3101 by making gene construct between (pCAMBIA1300- SiSAP4-GFP) and control (pCAMBIA1300-GFP). This was kept for incubation at $25^{\circ} \mathrm{C}$ with a $16 / 8 \mathrm{~h}$ (light/dark) photoperiod for $2 \mathrm{~d}$ and fluorescence signals were checked by confocal laser scanning microscope.

\section{Generation of Arabidopsis transgenic plants}

The full-length cDNA of SiSAP4 gene was inserted between $X b a \mathrm{I}$ and $K p n \mathrm{I}$ position (forward primer, 5'TGCTCTAGAATGGAACACAAGGAGGCG -3’; reverse primer, 5'- CGGGGTACCGATCTTGTCGAGCTTCTC 3', XbaI and KpnI sites underlined) of the pCAMBIA1300 vector. Prepared gene construct was inserted into GV3101 strain of Agrobacterium tumefaciens and then transformed into Arabidopsis using floral infiltration (Clough and Bent 1998). After hygromycin resistance screening and PCR detection, $\mathrm{T}_{3}$ homozygous transgenic lines were obtained and three lines were randomly selected for subsequent experiments. 


\section{Stress tolerance assays of transgenic plants}

To observe the effect of osmotic stress on transgenic Arabidopsis, 7-d-old seedlings were transplanted to MS medium with 0 or $250 \mathrm{mM}$ mannitol for $10 \mathrm{~d}$ and then phenotypes were observed. The primary root length of five transgenic plants and control (WT) were calculated. To further investigate the drought stress tolerance of transgenic Arabidopsis, 7-d-old seedlings were grown in plates filled with mixture of soil, well-watered and kept in growth chamber under short day conditions (12/12 light/dark) without watering. After a water-withholding period and then re-watering for $3 \mathrm{~d}$, the survival rate of transgenic plants and control (WT) were calculated. The abiotic stress-related physiological characterization of transgenic Arabidopsis, including cell membrane stability (CMS) and water loss rate was measured (Mao et al. 2010).

\section{Expression analysis of the stress response genes}

The expression level of stress response genes in transgenic Arabidopsis was analyzed by qRT-PCR. Arabidopsis seedlings were subjected to the MS medium with $250 \mathrm{mM}$ mannitol for $3 \mathrm{~h}$ and the tissue samples were harvested. Based on the conserved regions of stress response genes, specific primers were designed to detect the expression level (Supplementary Table S1).

\section{Transcriptional activity assay}

For transcriptional activity assays GAL4-based Matchmaker Two-Hybrid System (Clontech) with AH109 strain of Saccharomyces cerevisiae were utilized. For cloning purpose, pGBKT7 vector was used by inserting full length ORF of SiSAP4 and two truncations to make fusion with GAL4-binding domain and then transformed to AH109 yeast strain and kept for culturing until optical density at $600 \mathrm{~nm}$ reach to 1.0. Later, the suspension was grown into $\mathrm{SD} /-\mathrm{Trp}$ and SD/-Trp/-His medium. An empty pGBKT7 vector was used as control.

\section{Statistical analysis}

Three replications of each sample were utilized for the experiments. The data represented is the mean \pm SD. To study normal and drought stress conditions parameters data were analyzed by two tailed Student's t-test method. The significant differences were represented at the level $P<0.05$ or $P<0.01$.

\section{Results}

\section{Isolation and sequence analysis of SiSAP4}

The target gene SiSAP4 was isolated from foxtail millet. The full length of SiSAP4 was 516 bp, encoding 171 amino acids. The predicted molecular weight was $18.21 \mathrm{kD}$, and the isoelectric point was 8.28. Protein structure prediction SiSAP4 contained an A20 and an AN1 domain. Sequence alignment showed that the amino acid sequences of the two domains of SiSAP4 were highly similar to those of other species (Fig. 1A). Phylogenetic evolutionary analysis showed that SiSAP4 can be classified in other monocotyledonous plants, including sorghum, Zea mays, rice, wheat and Brachypodium distachyon (Fig. 1B).

\section{Expression patterns of SiSAP4 in various tissues and under abiotic stresses}

The expression patterns of SiSAP4 in various tissues at seedling stage were examined by qRT-PCR. The transcript of SiSAP4 was identified in leaf, stem and root. The highest expression levels were observed in leaf tissues. The lowest expression was checked in root (Fig. 2A). Hence, the current research mainly focused on the leaf tissues for the consequent analyses.

Expression levels of SiSAP4 detected under different stress treatments, including PEG, salt and cold showed that its expression was significantly activated by PEG, but relatively slightly by salt and cold (Fig. 2D). Under PEG conditions, the expression levels of SiSAP4 reached a peak at $1 \mathrm{~h}$ and maintained to $3 \mathrm{~h}$, with the corresponding maxima being 4.2 greater than the control. Under high salinity conditions, the expression levels of SiSAP4 firstly decreased and then increased gradually, reaching a peak at 9 h, 1.5 greater than the control. At low temperature, the expression of SiSAP4 showed two small peaks at $0.5 \mathrm{~h}$ and $12 \mathrm{~h}$, both 1.5 times higher than the control.

\section{Subcellular localization of SiSAP4}

Subcellular localization of SiSAP4 was observed in tobacco leaves. The construct SiSAP4-GFP fusing protein was driven by the CaMV $35 \mathrm{~S}$ promoter. The green fluorescence was observed in the cytoplasm, cell membrane and nucleus in tobacco leaf epidermal cells using fluorescence microscopy. Hence, it was found that SiSAP4-GFP was located in the cytoplasm, cell membrane and nucleus (Fig. 3).

\section{Overexpression of SiSAP4 in Arabidopsis to enhanced drought stress tolerance}

Three transgenic homozygous T3 Arabidopsis lines were selected randomly to study the role of SiSAP4 under abiotic stress. Variable level of expression observed in SiSAP4 transgenic lines though it was remarkable higher than expression in WT plants (Fig. 4B). The expression level of SiSAP4 was significantly up-regulated under osmotic stress. The 7-day-old transgenic Arabidopsis and WT were placed in MS medium with $250 \mathrm{~m} M$ mannitol. After 10 days, the phenotype of transgenic Arabidopsis and WT was basically the same in MS medium. The growth of transgenic 
A
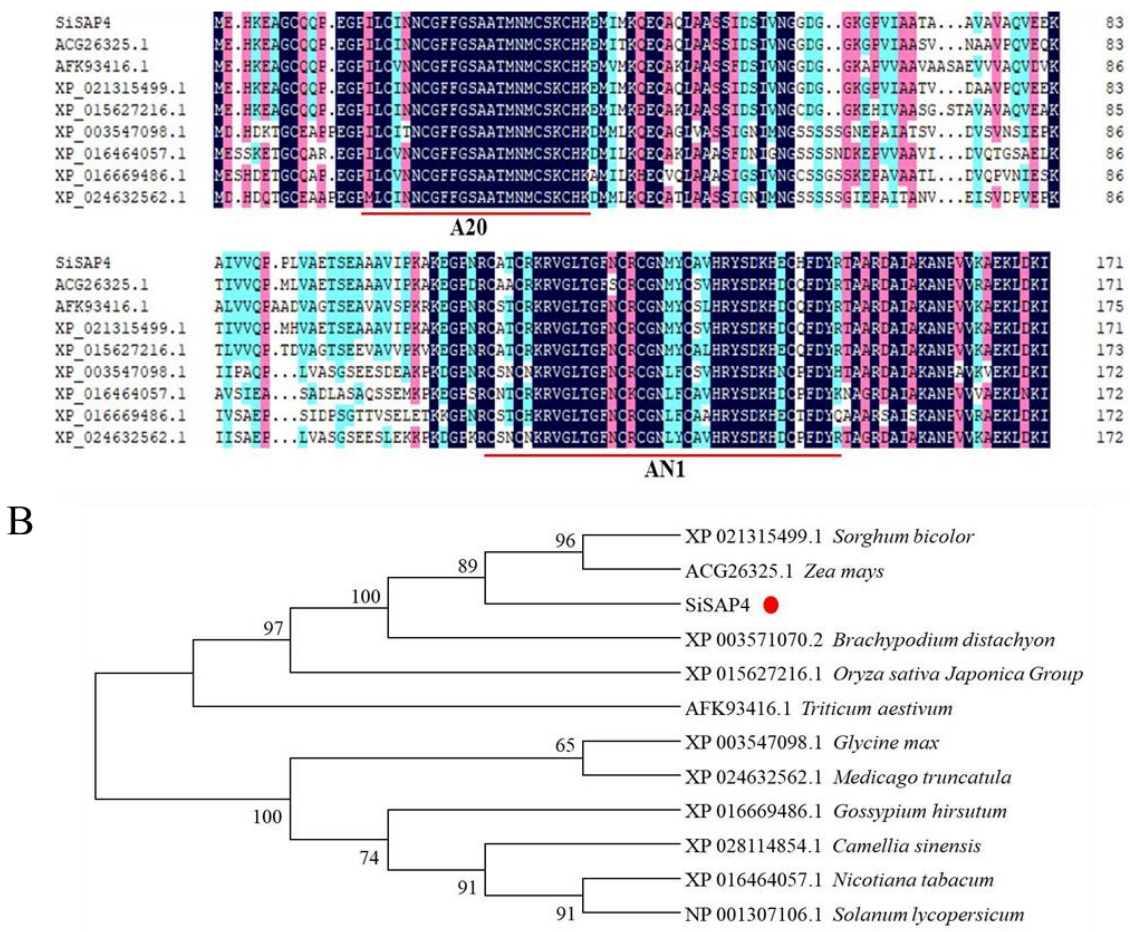

Fig. 1: Sequence alignment of SiSAP4 and SAPs in various plant species. (A) Alignment of SAPs from different plant species. ACG26325.1 from Zea mays, AFK93416.1 from Triticum aestivum, XP_021315499.1 from Sorghum bicolor, XP_015627216.1 from Oryza sativa Japonica Group, XP_003547098.1 from Glycine max, XP_016464057.1 from Nicotiana tabacum, XP_016669486.1 from Gossypium hirsutum, XP_024632562.1 from Medicago truncatula. Common identical amino acid residues are shaded black. The conserved A20 domain and AN1 domain are marked under the alignment with lines. (B) Construction phylogenetic tree of SAPs using neighbor-joining method with 1000 bootstrap replicates by MEGA5.0. SiSAP4 is marked with red dots

A

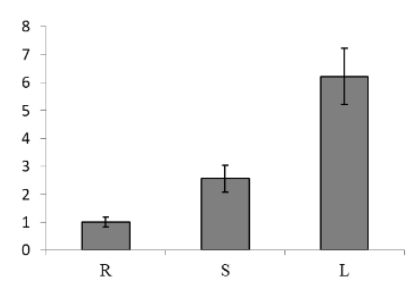

$\mathrm{C}$

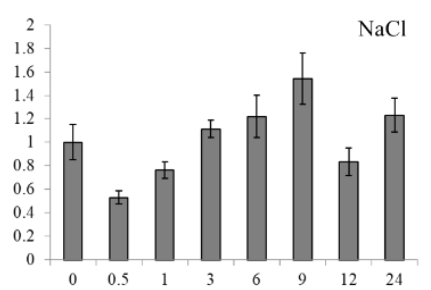

B

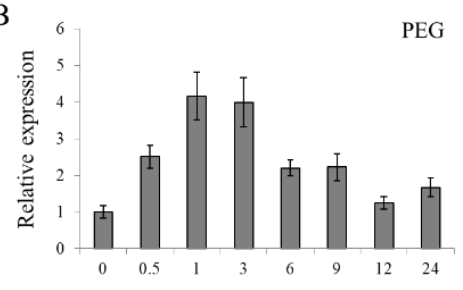

D

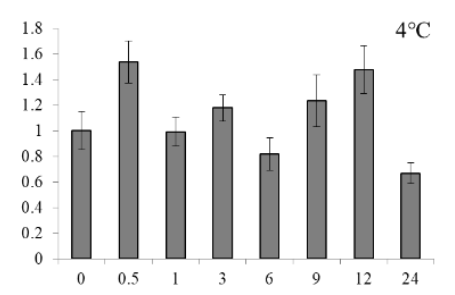

Fig. 2: Expression patterns of SiSAP4. (A) Tissue expression patterns of SiSAP4 at seedling stage. L, leaf; R, root; S, stem. (B) Relative expression of SiSAP4 under polyethlene glycol-6000 (PEG) treatment. (C) Relative expression of SiSAP4 under NaCl treatment. (D) Relative expression of SiSAP4 under cold $\left(4^{\circ} \mathrm{C}\right)$ treatment. Means were calculated from three independent experiments

Arabidopsis and WT both were inhibited in MS medium with $250 \mathrm{mM}$ mannitol, however, the growth of transgenic Arabidopsis was less inhibited (Fig. 4A). The length of primary root of transgenic Arabidopsis was significantly higher than WT (Fig. 4C).

To further verify the function of SiSAP4 under drought stress, the 7-day-old transgenic Arabidopsis and WT were planted in nutrient soil for drought stress treatment. Seedling 


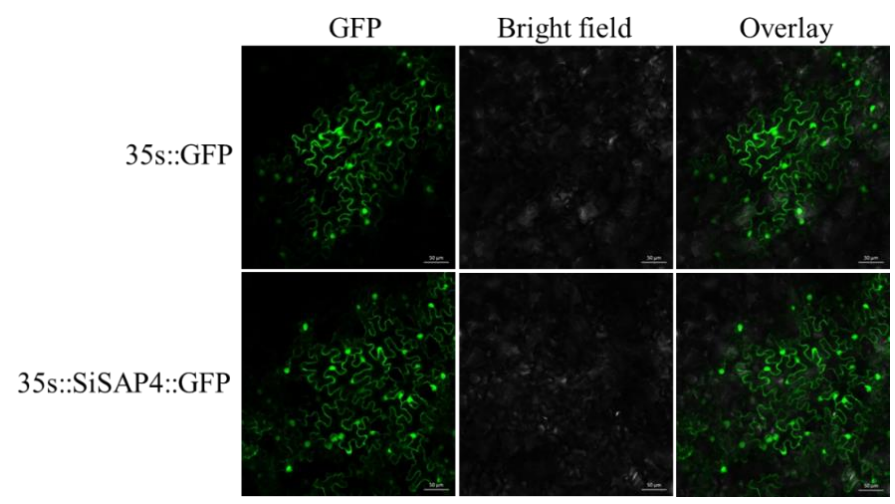

Fig. 3: Subcellular localization of SiSAP4 in tobacco leaf cells. The fusion construct (35S::SiSAP4::GFP) was transiently expressed in tobacco epidermal cells. Empty vector (35S::GFP) was used as control. Scale bar $=50 \mu \mathrm{m}$
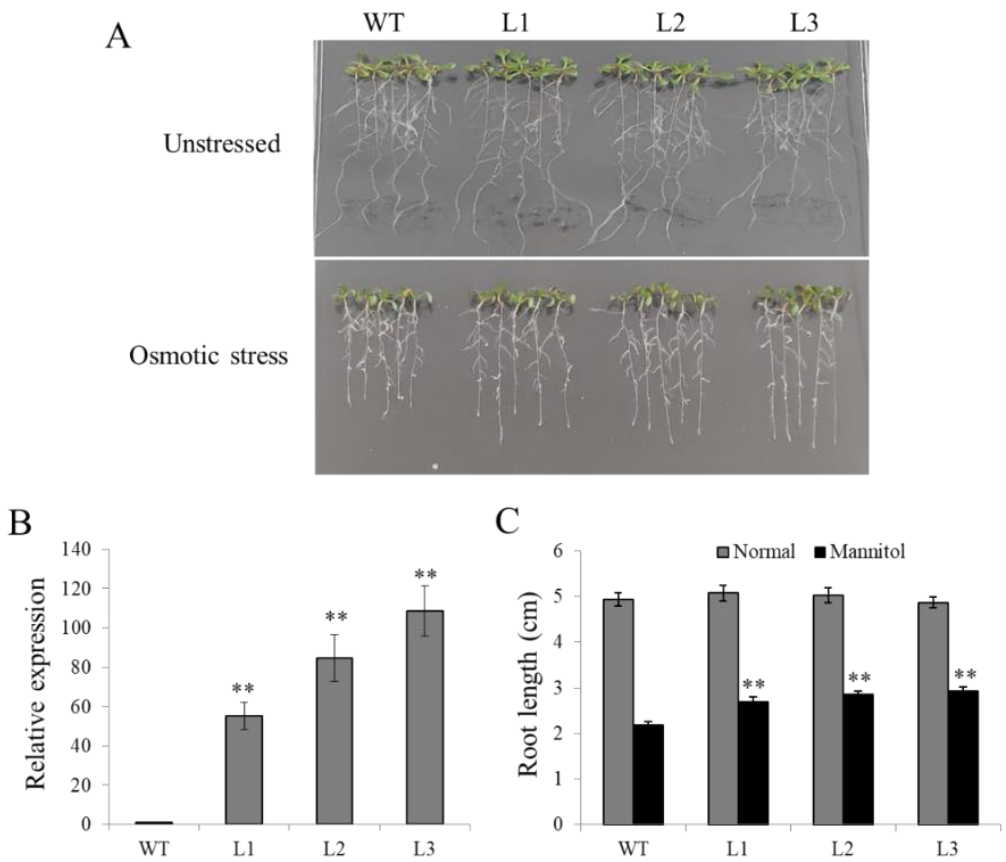

Fig. 4: Overexpression of SiSAP4 improves osmotic stress tolerance in transgenic Arabidopsis. (A) Phenotypes of three SiSAP4 transgenic lines (L1-3) and wild type (WT) under osmotic stress. (B) The expression level of SiSAP4 in three overexpressing Arabidopsis lines. (C) Comparison of primary root lengths of SiSAP4 overexpressing Arabidopsis lines. **, $P<0.01$

survival percentage was calculated after rewatering for three days, and it was significantly higher (56-69\%) for transgenic lines than WT $(17 \%)$ (Fig. 5A-B). There are certain physiological changes observed in plants due to drought stress. CMS of SiSAP4 transgenic plants was observed to be higher than WT (Fig. 5C). Water loss assay was performed in 8-h detached-rosette of 4 weeks old SiSAP4-transgenic overexpressed Arabidopsis plants and WT. The higher water loss rate was observed in WT than the transgenic plants (Fig. 5D).

\section{SiSAP4 enhances expression of abiotic stress response genes in transgenic plants}

Phenotypes assays indicated that SiSAP4 transgenic lines had enhanced tolerance to drought stress. Plants established intricate cellular signaling mechanism to manage the drought stress. In this study, it was further confirmed that several abiotic stress-responsive genes were up-regulated by SiSAP4. The expression level was checked in 8 abiotic stress related genes (P5CS1, RD29A, RD29B, RD22, COR47, COR15a, RAB18 and KIN1) under drought stress and normal environment. It was inferred that the expression level upregulated in P5CS1, RD29A, RD29B, RD22, COR47 and KIN1 genes under water deficit conditions in transgenic plants by 1.3 to 6.9 fold than WT plants. However, compared to the WT, expression level of transgenic plants in COR15a and $R A B 18$ genes had no remarkable changes under both water deficit and normal environment (Fig. 6). 


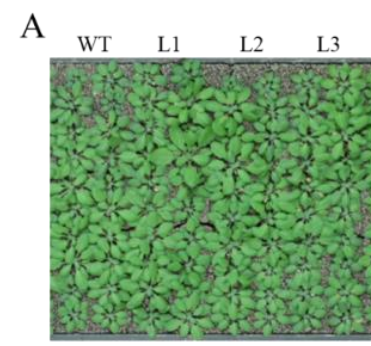

Before drought stress

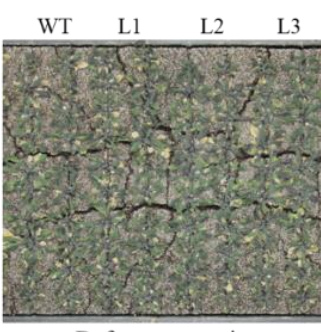

Before rewatering

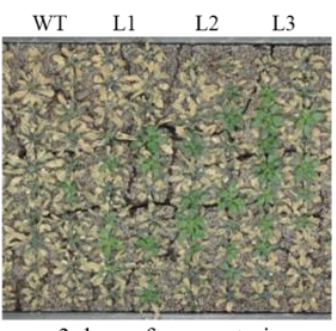

3 days after rewatering

B

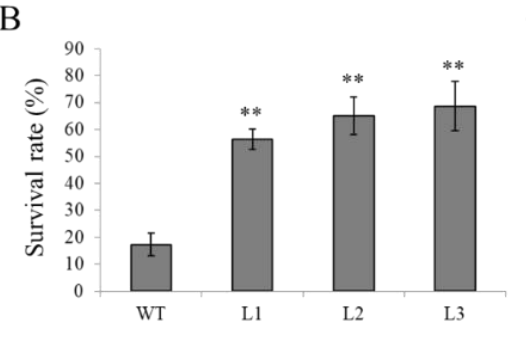

$\mathrm{C}$

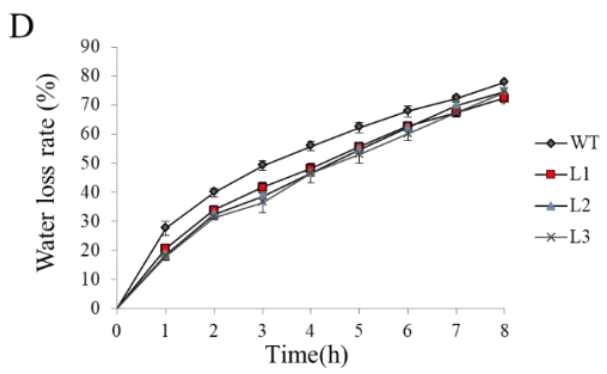

Fig. 5: Overexpression of SiSAP4 improves drought stress tolerance in transgenic Arabidopsis. (A) Phenotypes of three SiSAP4 transgenic lines (L1-3) and wild type (WT) under drought stress. Three transgenic lines and WT were planted in two rows, respectively. (B) Comparison of seedling survival rate between transgenic plants and WT. (C) Comparison of cell membrane stability (CMS) between transgenic plants and WT. (D) Comparison of water loss rates for detached rosettes between transgenic plants and WT. **, $P<0.01$

\section{SiSAP4 lacks transcriptional activation potential}

To detect the transcriptional activation of SiSAP4, the fulllength ORF of SiSAP4 and two truncations, according to the two domains (A20 and AN1), were cloned into pGBKT7 to produce in-frame fusions to GAL4-binding domain. The yeast containing two truncations and the full-length ORF of SiSAP4 can grow in SD-Trp medium, however, they don't grow in SD-Trp/-His medium (Fig. 7). The above results showed that SiSAP4 and two domains did not have transcriptional activation.

\section{Discussion}

There are abundant combinations of the domains of stress associated proteins (SAPs) in plants, including A20+AN1, A20, AN1 and 2AN1 etc. The most common combination of the domains was A20+AN1 (Vij and Tyagi 2008). In this study, SiSAP4 included an A20 and an AN1 domain, being a typical domain combination of SAP. In different plants, most members of the SAP gene family had no introns (Jain et al. 2008). SiSAP4 also had no introns, suggesting that it can be rapidly transcripted and expressed to perform biological functions.

Numerous studies show that the plant SAP gene family is constitutively expressed. For example, the transcript of TaSAP17-D had been shown to be expression in various tissues at different development stages and higher expression in leaves at seedling stage (Xu et al. 2018). In this study, expression analysis in different tissues at seedling stage showed that the expression levels of SiSAP4 were higher in leaves than in stems and roots, suggesting that it mainly played its biological role in leaves. The active and variable expression levels of SiSAP4 under different abiotic stresses were assessed. SiSAP4 was up-regulated in dehydration stress, suggesting that it played a major role in coping with the drought stress rather than salt and cold stresses. Most members of the SAP gene family were reported as positive regulators in the plant drought stress responses, such as AlSAP, MtSAPl and MusaSAPI (Saad et al. 2012; Sreedharan et al. 2012; Charrier et al. 2013). It was observed that SiSAP4 overexpressing plants possessed longer roots in the osmotic stress. Meanwhile, survival percentage of transgenic plants was improved in drought 


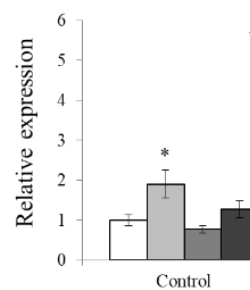

P5CS1
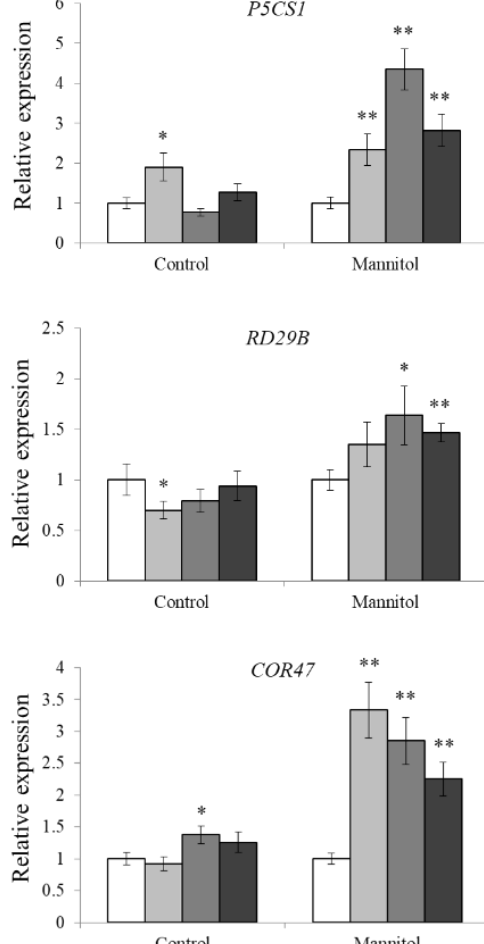

COR47
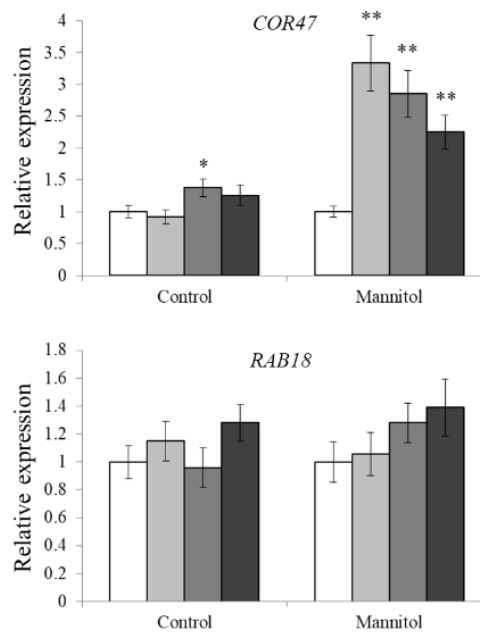
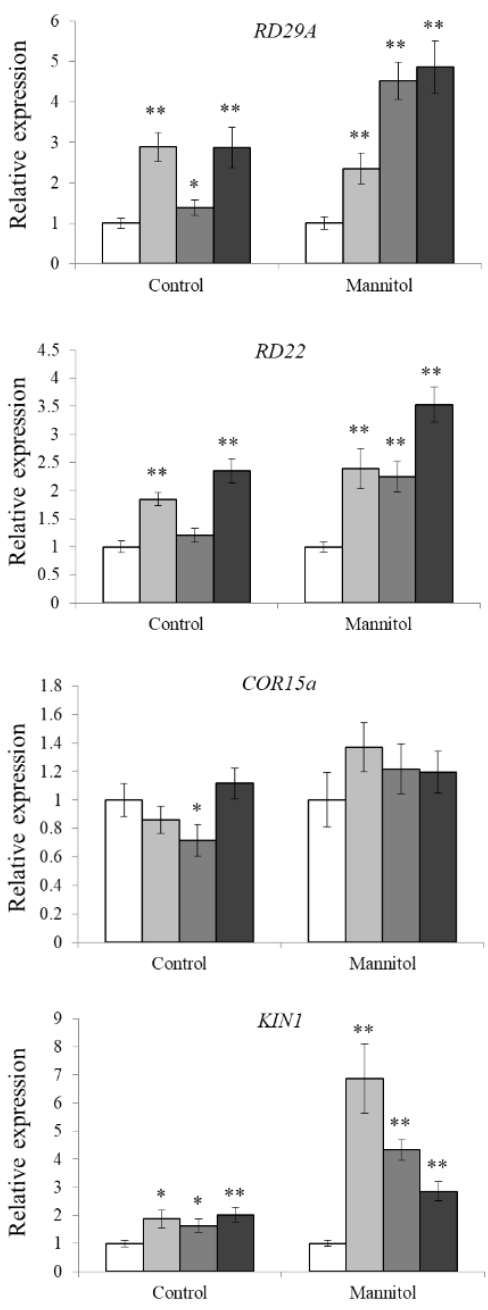

口WT ㅁL1 L2 घL3

Fig. 6: Effect of overexpression of SiSAP4 in transgenic Arabidopsis on the expression of stress-responsive genes. Relative expression levels of stress-responsive genes were determined by qRT-PCR in transgenic plants and wild type (WT) under normal condition and osmotic stress. *, $P<0.05 ; * *, P<0.01$

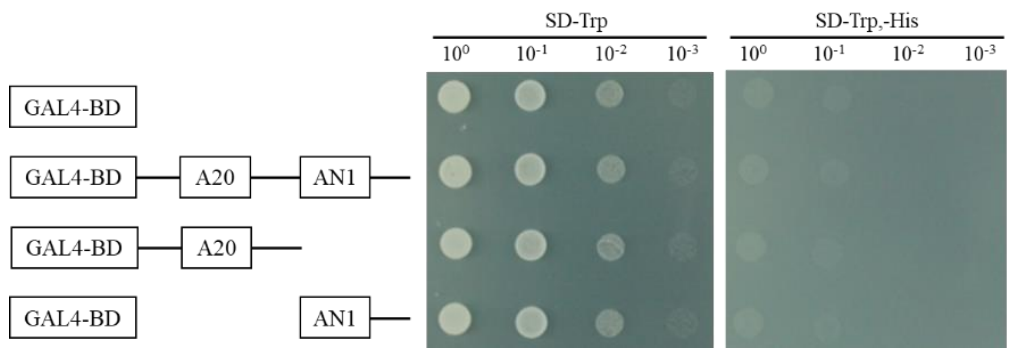

Fig. 7: Transcriptional activation activity assay of SiSAP4. According to the amino acid position of the conserved domain, transcriptional activation activity of SiSAP4 of the full-length and two truncations. A20 domain truncation is 1-111 amino acid residues. AN1 domain truncation is 112-171 amino acid residues

stress contrast to the WT plants. Drought stress induces various biochemical and physiological change. CMS and water loss rate were selected to monitor drought stress tolerance in the present study. Plants with higher CMS often have enhanced tolerance to drought stress (Farooq and
Azam 2006). It was inferred from the study that SiSAP4 transformants showed higher CMS under drought stress than the WT. Detached-leaf water loss rate is suggested as an indicator of water status (Clarke et al. 1989; Dhanda and Sethi 1998). In present research work the WT plants showed 
higher detached-leaf water loss rate than SiSAP4 transgenic plants, that strongly shows that transgenic plants had higher water retention capacity.

Plant signaling system during stress response was quite complex and mostly interconnected. Drought is a major abiotic stress to limit crop yields. Drought-related transcription factors can activate the expression of many downstream genes about regulation and signal transduction to improve plant stress resistance (Bartels and Sunkar 2005). The present studies show that SAPs can regulate the expression of downstream genes, especially those involved in stress responses (Wang et al. 2016). In this study, the overexpression of SiSAP4 upregulated a broad range of stress responsive genes. It is inferred that the improved tolerances to drought stress are major attributable to consistently and significantly enhanced expression of stress responsive genes including $P 5 C S 1, R D 29 A, R D 29 B, R D 22$, COR47 and KIN1. In contrast, no deviation in the expression of $C O R 15 a$ and $R A B 18$ was found in transgenic plants and WT suggesting the two genes are not involved in the drought stress pathway affected by SiSAP4. Taken together, SiSAP4 can be used as a candidate gene to play a role in crop resistance to drought stress. Interestingly, we found that SiSAP4 lacks transcriptional activation potential. It was speculated that SiSAP4 could work with other proteins to activate the expression of many downstream genes.

\section{Conclusion}

According to our findings, we suggested that the improved tolerance to drought stress by SiSAP4 overexpressing plants was due to the up-regulation expression of abiotic stress related genes. The SiSAP4 gene could be instrumental in improving drought stress tolerance in foxtail millet and other plants.

\section{Acknowledgements}

This work was supported by the System of National Modern Agriculture Technology (CARS-06-13.5-A28).

\section{Author Contributions}

Wenlu Li and Liguang Zhang designed and conducted the experiments; Faheeda Soomro and Pingyi Guo analyzed the experimental data; Xiangyang Yuan and Yixue Wang helped conceiving the study and participated in manuscript writing.

\section{References}

Baek D, JY Cha, S Kang, B Park, HJ Lee, H Hong, HJ Chun, DH Kim, MC Kim, SY Lee, DJ Yun (2015). The Arabidopsis a zinc finger domain protein ARS1 is essential for seed germination and ROS homeostasis in response to ABA and oxidative stress. Front Plant Sci 6; Article 963

Bartels D, R Sunkar (2005). Drought and salt tolerance in plants. Crit Rev Plant Sci 24:23-58
Chakraborty N, N Singh, K Kaur, N Raghuram (2015). G-protein signaling components GCR1 and GPA1 mediate responses to multiple abiotic stresses in Arabidopsis. Front Plant Sci 6; Article 1000

Charrier A, E Lelievre, AM Limami, E Planchet (2013). Medicago truncatula stress associated protein 1 gene (MtSAPl) overexpression confers tolerance to abiotic stress and impacts proline accumulation in transgenic tobacco. J Plant Physiol 170:874-877

Charrier A, E Planchet, D Cerveau, C Gimeno-Gilles, I Verdu, AM Limami, E Lelievre (2012). Overexpression of a Medicago truncatula stress-associated protein gene (MtSAPl) leads to nitric oxide accumulation and confers osmotic and salt stress tolerance in transgenic tobacco. Planta 236:567-577

Clarke JM, I Romagosa, S Jana, JP Srivastava, TN Mccaig (1989). Relationship of excised-leaf water loss rate and yield of durum wheat in diverse environments. Can J Plant Sci 69:1075-1081

Clough SJ, AF Bent (1998). Floral dip: A simplified method for Agrobacterium-mediated transformation of Arabidopsis thaliana. Plant J 16:735-743

Dhanda SS, GS Sethi (1998). Inheritance of excised-leaf water loss and relative water content in bread wheat (Triticum aestivum). Euphytica 104:39-47

Farooq S, F Azam (2006). The use of cell membrane stability (CMS) technique to screen for salt tolerant wheat varieties. J Plant Physiol 163:629-637

Giri J, PK Dansana, KS Kothari, G Sharma, S Vij, AK Tyagi (2013). SAPs as novel regulators of abiotic stress response in plants. Bioessays 35:639-648

Han GL, CX Lu, JR Guo, ZQ Qiao, N Sui, NW Qiu, BS Wang (2020). Corrigendum: $\mathrm{C} 2 \mathrm{H} 2$ zinc finger proteins: master regulators of abiotic stress responses in plants. Front Plant Sci 11; Article 298

Hirayama T, K Shinozaki (2010). Research on plant abiotic stress responses in the post-genome era: Past, present and future. Plant $J$ 61:1041-1052

Hozain Md, H Abdelmageed, J Lee, M Kang, M Fokar, RD Allen, AS Holaday (2012). Expression of AtSAP5 in cotton up-regulates putative stress-responsive genes and improves the tolerance to rapidly developing water deficit and moderate heat stress. J Plant Physiol 169:1261-1270

Jain M, P Khurana, AK Tyagi, JP Khurana (2008). Genome-wide analysis of intronless genes in rice and Arabidopsis. Funct Integr Genom 8:69-78

Kanneganti V, AK Gupta (2008). Overexpression of OsiSAP8, a member of stress associated protein (SAP) gene family of rice confers tolerance to salt, drought and cold stress in transgenic tobacco and rice. Plant Mol Biol 66:445-462

Mao XG, HY Zhang, SJ Tian, XP Chang, RL Jing (2010). TaSnRK2.4, an SNF1-type serine/threonine protein kinase of wheat (Triticum aestivum L.), confers enhanced multistress tolerance in Arabidopsis. $J$ Exp Bot 61:683-696

Min DH, FY Xue, YN Ma, M Chen, ZS Xu, LC Li, XM Diao, GQ Jia, YZ Ma (2013). Characteristics of $P P 2 C$ gene family in foxtail millet (Setaria italica). Acta Agron Sin 39:2135-2144

Mukhopadhyay A, S Vij, AK Tyagi (2004). Overexpression of a zincfinger protein gene from rice confers tolerance to cold, dehydration, and salt stress in transgenic tobacco. Proc Natl Acad Sci USA 101:6309-6314

Muthamilarasan M, M Prasad (2015). Advances in Setaria genomics for genetic improvement of cereals and bioenergy grasses. Theor Appl Genet 128:1-14

Saad RB, D Fabre, D Mieulet, D Meynard, M Dingkuhn, A Al-Doss, E Guiderdoni, A Hassairi (2012). Expression of the Aeluropus littoralis AlSAP gene in rice confers broad tolerance to abiotic stresses through maintenance of photosynthesis. Plant Cell Environ 35:626-643

Saad RB, N Zouari, WB Ramdhan, J Azaza, D Meynard, E Guiderdoni, A Hassairi (2010). Improved drought and salt stress tolerance in transgenic tobacco overexpressing a novel A20/AN1 zinc-finger "AlSAP" gene isolated from the halophyte grass Aeluropus littoralis. Plant Mol Biol 72:171-190

Schmittgen TD, KJ Livak (2008). Analyzing real-time PCR data by the comparative C(T) method. Nat Protoc 3:1101-1108 
Shi HT, C Jiang, TT Ye, DX Tan, RJ Reiter, H Zhang, RY Liu, ZL Chan (2015). Comparative physiological, metabolomic, and transcriptomic analyses reveal mechanisms of improved abiotic stress resistance in Bermuda grass [Cynodon dactylon (L). Pers.] by exogenous melatonin. J Exp Bot 66:681-694

Solanke AU, MK Sharma, AK Tyagi, AK Sharma (2009). Characterization and phylogenetic analysis of environmental stress-responsive SAP gene family encoding A20/AN1 zinc finger proteins in tomato. Mol Genet Genomics 282:153-164

Sreedharan S, UKS Shekhawat, TR Ganapathi (2012). MusaSAP1, a A20/AN1 zinc finger gene from banana functions as a positive regulator in different stress responses. Plant Mol Biol 80:503-517

Tamura K, D Peterson, N Peterson, G Stecher, M Nei, S Kumar (2011). MEGA5: Molecular evolutionary genetics analysis using maximum likelihood, evolutionary distance, and maximum parsimony methods. Mol Biol Evol 28:2731-2739

Vij S, AK Tyagi (2008). A20/AN1 zinc-finger domain-containing proteins in plants and animals represent common elements in stress response. Funct Integr Genom 8:301-307

Wang F, RA Coe, S Karki, S Wanchana, V Thakur, A Henry, HC Lin, J Huang, S Peng, WP Quick (2016). Overexpression of OsSAP16 regulates photosynthesis and the expression of a broad range of stress response genes in rice (Oryza sativa L.). PLoS One 11; Article e0157244

Wang K, YF Ding, C Cai, ZX Chen, C Zhu (2019). The role of C2H2 zinc finger proteins in plant responses to abiotic stresses. Physiol Plantarum 165:690-700
Wang YH, LR Zhang, LL Zhang, T Xing, JZ Peng, SL Sun, G Chen, XJ Wang (2013). A novel stress-associated protein SbSAP14 from Sorghum bicolor confers tolerance to salt stress in transgenic rice. Mol Breed 32:437-449

Xu QF, XG Mao, YX Wang, JY Wang, YJ Xi, RL Jing (2018). A wheat gene TaSAP17-D encoding an AN1/AN1 zinc finger protein improves salt stress tolerance in transgenic Arabidopsis. J Integr Agric 17:507-516

Xuan N, Y Jin, HW Zhang, YH Xie, YJ Liu, GY Wang (2011). A putative maize zinc-finger protein gene, ZmAN13, participates in abiotic stress response. Plant Cell Tiss Org 107:101-112

Yang ZR, HS Zhang, XK Li, HM Shen, JH Gao, SY Hou, B Zhang, S Mayes, M Bennett, JX Ma, CY Wu, Y Sui, YH Han, XC Wang (2020). A mini foxtail millet with an Arabidopsis-like life cycle as a C4 model system. Nat Plant 6:1167-1178

Zhang H, YP Liu, F Wen, DM Yao, L Wang, J Guo, L Ni, AY Zhang, MP Tan, MY Jiang (2014). A novel rice C2H2-type zinc finger protein, ZFP36, is a key player involved in abscisic acid-induced antioxidant defenceand oxidative stress tolerance in rice. J Exp Bot 65:5795-5809

Zhang L, YZ Li, WJ Lu, F Meng, CA Wu, XQ Guo (2012). Cotton GhMKK5 affects disease resistance, induces HR-like cell death, and reduces the tolerance to salt and drought stress in transgenic Nicotiana benthamiana. J Exp Bot 63:3935-3951

Zhang N, YJ Yin, XY Liu, SM Tong, JW Xing, Y Zhang, RN Pudake, EM Izquierdo, HR Peng, MM Xin, ZR Hu, ZF Ni, QX Sun, YY Yao (2017). The E3 ligase TaSAP5 alters drought stress responses by promoting the degradation of DRIP proteins. Plant Physiol 175:1878-1892 